\title{
CARACTERÍSTICAS DE LA ISLA DE CALOR URBANA EN BARCELONA (NE ESPAÑA) EN EL PERIODO 2006-2017
}

\section{CHARACTERISTICS OF THE URBAN HEAT ISLAND IN BARCELONA (NE SPAIN) ALONG THE 2006-2017 PERIOD}

\author{
MARTÍNEZ, Maria Dolors \\ Universidad Politécnica de Catalunya (UPC) \\ Departamento de Física (FIS), Centro de Política de Suelo y Valoraciones (CPSV) \\ Profesora Titular de Universidad \\ Av. Diagonal 649, 08028. Barcelona, España \\ Correo electrónico: dolors.martinez@upc.edu \\ Teléfono: +34 934016378
}

LANA, Xavier

Universidad Politécnica de Catalunya (UPC)

Departamento de Física (FIS)

Profesor Titular de Universidad

Av. Diagonal 647, 08028. Barcelona, España

Correo electrónico: francisco.javier.lana@upc.edu

Teléfono: +34 934010825

\section{SERRA, Carina}

Universidad Politécnica de Catalunya (UPC)

Departamento de Física (FIS)

Profesora Titular de Universidad

Av. Diagonal 647, 08028. Barcelona, España

Correo electrónico: carina.serra@upc.edu

Teléfono: +34 934015934

ROCA, Josep

Universidad Politécnica de Catalunya (UPC)

Departamento de Tecnología de la Arquitectura (TA), Centro de Política de Suelo y Valoraciones (CPSV) Catedrático de Universidad

Av. Diagonal 649, 08028. Barcelona, España

Correo electrónico: josep.roca@upc.edu

Teléfono: +34 934016396

\section{ARELLANO, Blanca}

Universidad Politécnica de Catalunya (UPC)

Departamento de Tecnología de la Arquitectura (TA), Centro de Política de Suelo y Valoraciones (CPSV)

Profesora asociada y Personal de Investigación (PSR)

Av. Diagonal 649, 08028 Barcelona, España

Correo electrónico: blanca.arellano@upc.edu

Teléfono: +34 934054383

\section{BIERE, Rolando}

Universidad Politécnica de Catalunya (UPC)

Departamento de Tecnología de la Arquitectura (TA), Centro de Política de Suelo y Valoraciones (CPSV) Profesor asociado y Personal de Investigación (PSR)

Av. Diagonal 649, 08028. Barcelona, España

Correo electrónico: rolando.biere@upc.edu

Teléfono: +34 934016396

Citación: MARTINEZ, M. D. et al. Características de la isla de calor urbana en Barcelona (NE España) en el periodo 20062017. En: Libro de proceedings, CTV 2018. XII Congreso Internacional Ciudad y Territorio Virtual. "Ciudades y Territorios Inteligentes". UNCuyo, Mendoza, 5-7 septiembre 2018. Barcelona: CPSV, 2018, p. 369-380. 


\section{MOIX, Montserrat}

Universidad Politécnica de Catalunya (UPC)

UTG Ámbito Arquitectura Barcelona (UTGAB)

Personal de investigación (PAS)

Av. Diagonal 649, 08028 Barcelona, España

Correo electrónico: monserrat.moix@upc.edu

Teléfono: +34 934010760

Palabras Clave: isla de calor urbana; Barcelona; temperaturas mínimas diarias; intensidad de la isla de calor; influencia de la velocidad del viento

Key words: urban heat island; Barcelona city center; daily minimum temperatures; UHI intensity; influence of wind speed

\section{Resumen}

El objetivo principal de este estudio es caracterizar la intensidad de la isla de calor urbana en la ciudad de Barcelona a lo largo del periodo octubre 2006 - octubre 2017. La intensidad de la isla de calor es la diferencia de temperatura, en un momento determinado, entre el núcleo urbano y las zonas no urbanas (o periurbanas) próximas. Es el indicador más sencillo para cuantificar el calentamiento de la ciudad, en relación a su entorno, especialmente durante las horas nocturnas. Para que la diferencia de temperaturas se pueda atribuir a los efectos de la urbanización, se requiere que los emplazamientos que se comparan sean suficientemente cercanos como para tener el mismo clima regional y que sus factores geográficos locales (altitud y distancia al mar) sean similares. En este estudio, se calculan, día a día, las diferencias de las temperaturas mínimas registradas en la estación meteorológica de El Raval, situada en el centro de Barcelona, y el observatorio meteorológico del aeropuerto de Barcelona (El Prat), situado en el área metropolitana. El aeropuerto no se ubica en una zona rural, pero es un área muy abierta, con un elevado factor de visión del cielo, por lo tanto, con un remarcable enfriamiento nocturno.

El periodo de estudio, que abarca 11 años, proporciona más de 4000 datos diarios de diferencias de temperatura mínima $\left(\Delta T_{\min }\right)$. Se analiza la distribución de frecuencias de los valores de $\Delta T_{\min }$ así como su valor máximo y medio, a escalas anual, semianual y estacional. Es de destacar que el $94.5 \%$ de las noches son más cálidas en la estación urbana (El Raval). Esta proporción asciende hasta el $98.4 \%$ durante el invierno. La diferencia máxima es de $7.1^{\circ} \mathrm{C}$ y, en promedio, las noches de invierno son $3.0^{\circ} \mathrm{C}$ más cálidas en El Raval. El análisis del espectro de potencia muestra la periodicidad anual y semianual de la intensidad de la isla de calor. La distribución de los valores de $\Delta T_{\min }$, en función del día del año, permite explorar la vinculación de la intensidad de la isla de calor con los periodos fríos o cálidos del año, especialmente, para los casos de los valores extremos de $\Delta T_{\min }$, y también para las escasas ocasiones en la noche es más fría en El Raval. Finalmente, se analiza el efecto de la velocidad del viento registrado en la estación urbana sobre los valores medio y máximo de $\Delta T_{\text {minn, }}$ dividiendo el rango de velocidades en intervalos de $0.2 \mathrm{~m} \cdot \mathrm{s}^{-1}$. Los resultados muestran claramente una disminución del valor medio de $\Delta T_{\min }$ al aumentar la velocidad del viento. Para los valores máximos de $\Delta T_{\min }$, se obtiene que es necesario superar un cierto valor crítico de la velocidad (alrededor de $2.5 \mathrm{~m} \cdot \mathrm{s}^{-1}$ ) para que el efecto del viento comience a atenuar los episodios más intensos de la isla de calor. 


\section{Abstract}

The main goal of this study is the characterization of the intensity of the urban heat island (UHI) in Barcelona city center along the October 2006-October 2017 period. The UHI intensity is evaluated by computing the difference in daily minimum temperatures, on a certain moment, between the city center and the peripheral or non-urban neighboring areas. It is the simplest indicator to quantify the warming of the city, in relation to the surrounding environment, especially at night. In order for the temperature differences can be attributed to the influence of urbanization, the sites to be compared must be nearby enough to have the same regional climate and their local geographical factors (altitude and distance from sea) be very similar. In this study, we compute the differences in daily minimum temperatures recorded at the meteorological station El Raval, in Barcelona downtown, and those recorded at the fix observatory of Barcelona Airport (EI Prat), located in the metropolitan area. Although the airport is not placed in a rural environment, it is a broadly open area, with a high sky view factor and, consequently, with a marked night cooling.

The recording period spans 11 years, thus providing more than 4000 daily data of minimum temperature differences $\left(\Delta T_{\min }\right)$. The frequency distribution of daily $\Delta T_{\min }$ and their maximum and average values are investigated at annual, half-annual and seasonal scales. It is worth remarking that $94.5 \%$ of nights are warmer at the urban site (El Raval), this percentage increasing until $98.4 \%$ in winter. The maximum temperature difference is $7.1^{\circ} \mathrm{C}$ and, on average, winter nights are $3.0^{\circ} \mathrm{C}$ warmer at El Raval. The power spectrum shows that UHI intensity has annual and half-annual periodicities. The distribution of $\Delta T_{\min }$ values along the calendar days permits exploring the linkages of $\mathrm{UHI}$ intensity to cold and hot periods of the year, especially, for the cases of extreme $\Delta T_{\min }$ values, and for the scarce colder nights at El Raval. Finally, the influence of wind velocity recorded at the urban site in average and maximum $\Delta T_{\min }$ is explored for different wind speed classes, at $0.2 \mathrm{~m} \cdot \mathrm{s}^{-1}$ intervals. A clear decrease of average $\Delta T_{\min }$ with increasing wind speed is observed. With respect to maximum $\Delta T_{\min }$ values, the results indicate that a certain critical wind speed value (around $2.5 \mathrm{~m} \cdot \mathrm{s}^{-1}$ ) has to be exceeded for an increase of wind velocity starts weakening the most intense UHI episodes.

\section{Introducción}

El estudio del fenómeno de la isla de calor urbana (UHI) viene recibiendo creciente atención en las últimas décadas. Aunque las áreas urbanas cubren una pequeña proporción de la superficie de la Tierra, aproximadamente un $54 \%$ de la población vive en zonas urbanas, y se espera que este número alcance el 66\% en 2050 (Naciones Unidas, 2014). Desde el primer análisis de este fenómeno, realizado para la ciudad de Londres (Howard, 1833), se han llevado a cabo numerosas investigaciones sobre los efectos de la modificación antropogénica de los usos del suelo y de las emisiones de calor en un gran número de ciudades y para todo tipo de zonas climáticas. Los cambios asociados a la urbanización conllevan una modificación local del clima de las ciudades (Wanner and Hertig, 1984), que viene influenciado, en cada caso, por factores relacionados con las características de la ciudad, como su tamaño, la densidad de la edificación, la geometría urbana y la distribución de los distintos usos del suelo (Oke, 1982). Las condiciones meteorológicas (situación sinóptica, nubosidad, velocidad del viento, humedad relativa) pueden contribuir a potenciar o atenuar el crecimiento de la isla de calor (Morris and Simmonds, 2000; Morris et al., 2001; Makar et al., 2006; László et al, 2016). La isla de calor suele alcanzar su mayor intensidad en largas noches invernales, con cielo despejado y viento en calma (Arnfield, 2003). Algunos estudios referentes al fenómeno de isla de calor en 
Barcelona y su área metropolitana son los de Moreno-García (1994), Martín-Vide et al. (2015b) y Arellano y Roca (2016), entre otros. En relación a otras ciudades del área mediterránea, podemos citar los estudios de Montávez et al. (2000), Giannaros and Melas (2012), Papanastasiou and Kittas (2012) y Founda et al. (2015), entre muchos otros.

La intensidad de la isla de calor se puede evaluar a través de la comparación entre las temperaturas registradas en un punto urbano y en un punto rural próximo (Figuerola et al., 1998; Fortuniak et al., 2006, entre otros). Es el indicador más sencillo que permite cuantificar el calentamiento de la ciudad, en relación a su entorno (Memon et al., 2009; Martín-Vide et al., 2015a). El objetivo principal de este trabajo es caracterizar la intensidad de la isla de calor urbana en la ciudad de Barcelona durante el periodo 2006-2017.

Para ello, se calcula la diferencia entre las temperaturas mínimas diarias registradas en la estación meteorológica El Raval, situada en el centro de la ciudad, en un punto muy cercano a la zona más cálida de la ciudad durante la noche (Martín-Vide et al., 2015b), y las temperaturas mínimas registradas en el aeropuerto de Barcelona (El Prat), situado en el área metropolitana. Se analiza la distribución de frecuencias de los valores de las diferencias diarias de temperatura mínima $\left(\Delta T_{\min }\right)$, así como su valor máximo y medio, a escalas anual, semianual y estacional. Se calcula el espectro de potencia para analizar la periodicidad de la intensidad de la isla de calor. La distribución de los valores de $\Delta T_{\min }$, en función del día del año, permite explorar la vinculación de la intensidad de la isla de calor con los periodos fríos o cálidos del año. Finalmente, se analiza el efecto de la velocidad del viento registrado en la estación El Raval sobre los valores medio y máximo de $\Delta T_{\min }$, para diferentes intervalos de velocidad del viento.

\section{Selección de los puntos de muestreo y base de datos}

La base de datos principal consiste en las temperaturas mínimas diarias registradas, durante el periodo octubre 2006-octubre 2017, en la estación meteorológica de El Raval y en el observatorio meteorológico del aeropuerto de Barcelona (El Prat). El periodo de estudio (11 años) proporciona más de 4000 datos diarios de diferencias de temperatura mínima $\left(\Delta T_{\min }\right)$. Con el propósito de explorar la influencia del viento en la intensidad de la isla de calor, se consideran también los datos diarios de velocidad del viento registrada en El Raval.

La Figura 1 muestra la localización de las dos estaciones. En las imágenes de satélite de la Figura 2, se muestra el entorno en el que se ubican ambas estaciones. En el caso de El Raval, es una zona urbana muy densamente edificada y poblada. El aeropuerto no está situado en un área rural, pero no es posible encontrar zonas realmente rurales suficientemente próximas a la ciudad de Barcelona como para hacer comparaciones válidas. Sin embargo, es un área muy abierta, con un elevado factor de visión del cielo, por lo que presenta un remarcable enfriamiento nocturno, en contraste con el del centro de Barcelona (Martín-Vide et al., 2015b).

En la Tabla 1 se presentan las coordenadas de las dos estaciones, sus factores geográficos (altitud sobre el nivel del mar y distancia a la costa) y los valores de NDVI (normalized difference vegetation index) y NDBI (normalized difference build-up index), índices que describen el grado de cobertura vegetal y de densidad de edificación, respectivamente. De acuerdo con Martín-Vide et al. (2015a), para que las diferencias de temperatura sean atribuibles a los efectos de la urbanización, se requiere que los puntos que se comparan tengan altitud y distancia al mar similares. Las diferencias de altitud no deben superar $30 \mathrm{~m} \mathrm{y}$, para ciudades costeras, las distancias al mar no deben diferir en más de $800 \mathrm{~m}$. Como muestra la Tabla 1, las dos estaciones seleccionadas cumplen estos requisitos. 
Figura 1. Localización de las estaciones meteorológicas El Raval y El Prat-Aeropuerto

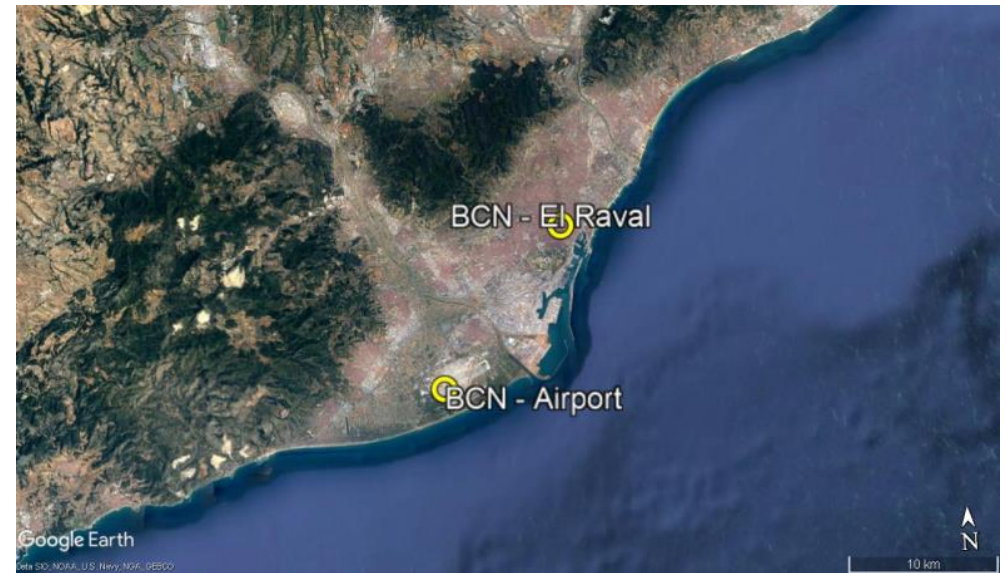

Fuente: Elaboración propia, sobre Google Maps

Figura 2. Entorno de las estaciones meteorológicas EI Raval y El Prat-Aeropuerto
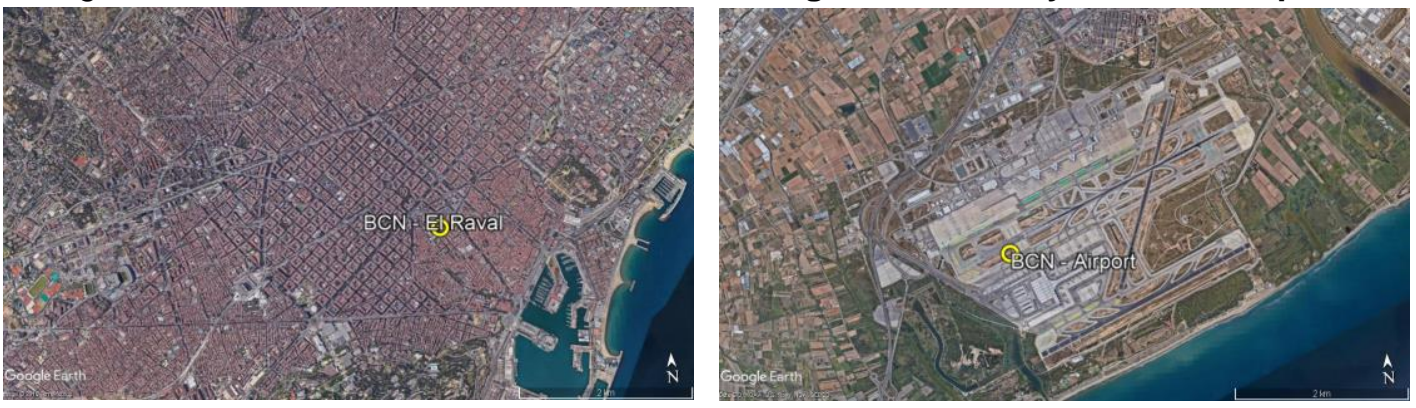

Fuente: Elaboración propia, sobre Google Maps

Tabla 1. Localización, factores geográficos y valores de los índices NDVI y NDBI de las dos estaciones meteorológicas

\begin{tabular}{|l|c|c|c|c|c|c|}
\hline Estación & Latitud & Longitud & Altitud (m) & Distancia al mar (m) & NDVI & NDBI \\
\hline El Raval & $41.3839^{\circ} \mathrm{N}$ & $2.1677^{\circ} \mathrm{E}$ & 33 & 1324 & 0.141 & 0.088 \\
\hline El Prat $(\mathrm{Ap})$ & $41.2928^{\circ} \mathrm{N}$ & $2.0700^{\circ} \mathrm{E}$ & 4 & 1802 & 0.145 & 0.049 \\
\hline
\end{tabular}

Fuente: Elaboración propia

\section{Metodología y resultados}

La intensidad diaria de la isla de calor se evalúa mediante la diferencia entre las temperaturas mínimas $\left(\Delta T_{\text {min }}\right)$, registradas en las estaciones de El Raval y El Prat (Ap). Un valor positivo de $\Delta T_{\min }$ indica, pues, una noche más cálida en El Raval que en El Prat (Ap).

\subsection{Distribución de frecuencias de las diferencias de temperatura mínima}

El histograma de la Figura 3 muestra la distribución, en clases de $1.0^{\circ} \mathrm{C}$, de las diferencias diarias en la temperatura mínima $\left(\Delta T_{\min }\right)$ entre El Raval y El Prat $(A p)$, para el periodo considerado (octubre 2006-octubre 2017), que comprende más de 4000 datos diarios. Es de destacar que el $94.5 \%$ de las diferencias son positivas, es decir, solo en un $5.5 \%$ de los días la temperatura mínima en el centro de Barcelona ha sido inferior a la de El Prat (Ap). La diferencia media de temperatura es de $2.0^{\circ} \mathrm{C}$ y la clase modal es la de $1.0-2.0^{\circ} \mathrm{C}$. La máxima diferencia de temperatura es de $7.1^{\circ} \mathrm{C}$ y se registró el 18/11/2007.

Citación: MARTINEZ, M. D. et al. Características de la isla de calor urbana en Barcelona (NE España) en el periodo 2006 2017. En: Libro de proceedings, CTV 2018. XII Congreso Internacional Ciudad y Territorio Virtual. "Ciudades y Territorios Inteligentes". UNCuyo, Mendoza, 5-7 septiembre 2018. Barcelona: CPSV, 2018, p. 369-380. 
Figura 3. Histograma de los valores de $\Delta T_{\min }$ para todo el periodo (Oct 2006-Oct 2017)

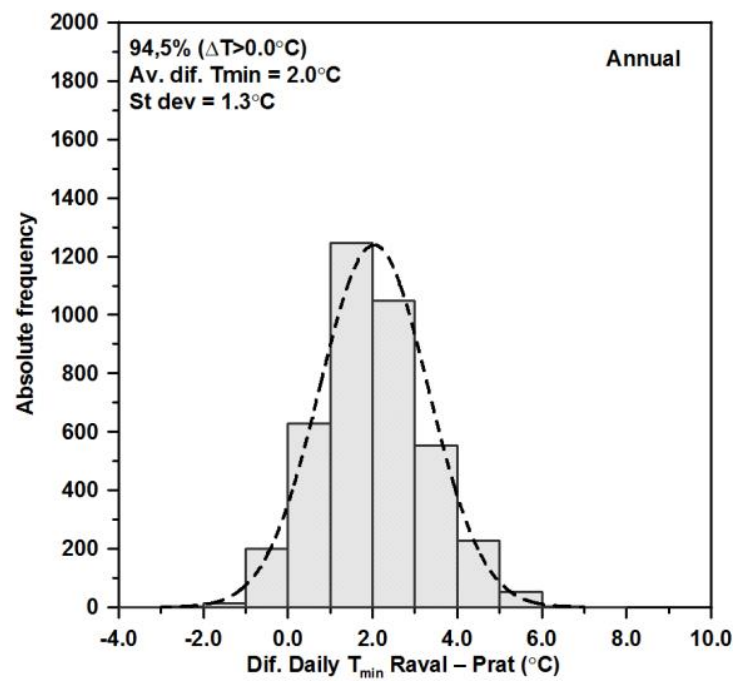

Fuente: Elaboración propia

Figura 4. Histograma de los valores de $\Delta T_{\min }$ a escala semianual
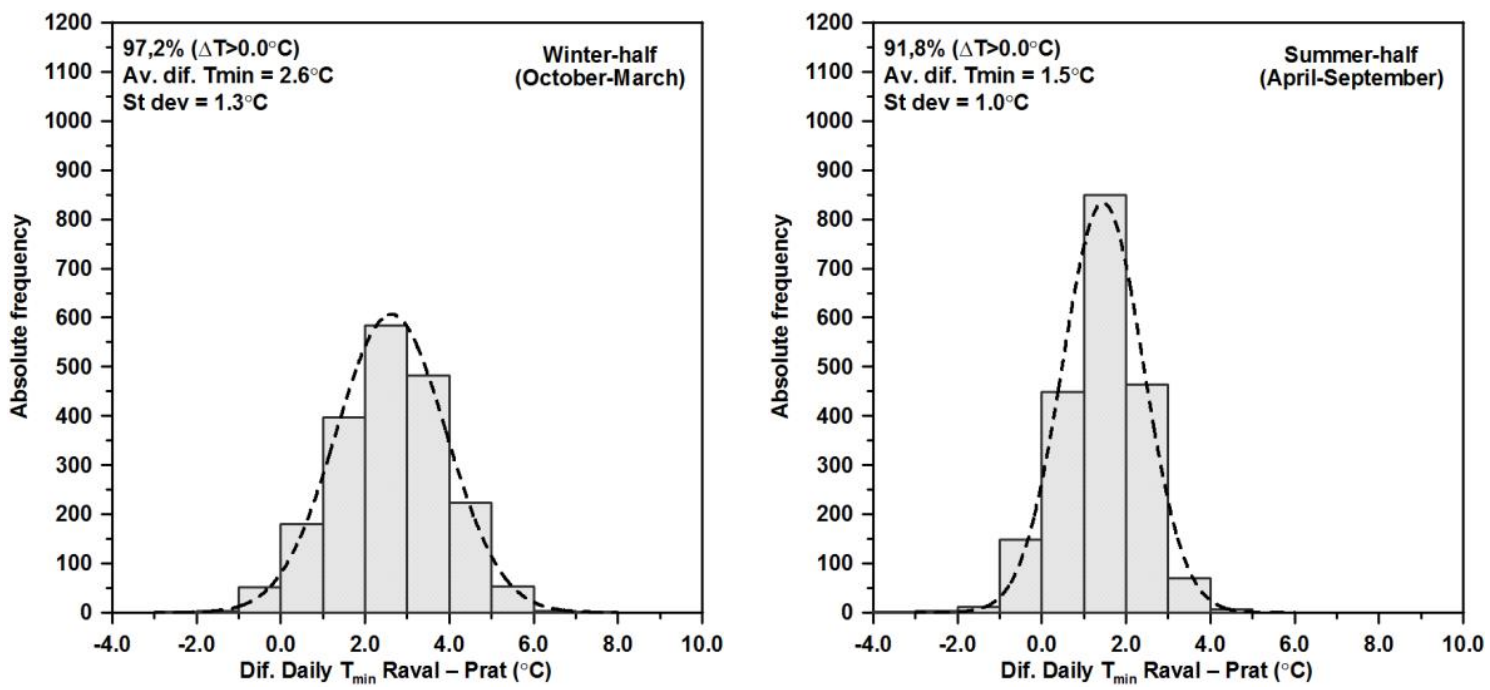

Fuente: Elaboración propia

Los histogramas de la Figura 4 muestran la distribución de los valores de $\Delta T_{\min }$ a escala semianual, es decir, distinguiendo entre la mitad más fría del año (de octubre a marzo) y la más cálida (de abril a septiembre).

Se observa que la isla de calor es más frecuente e intensa en el periodo frío, en el que un $97.2 \%$ de las noches son más cálidas en El Raval, con una diferencia media de temperatura de $2.6^{\circ} \mathrm{C}$. La clase modal es la de $2.0-3.0^{\circ} \mathrm{C}$. Todas las diferencias extremas de temperatura (5.0$7.0^{\circ} \mathrm{C}$ ) corresponden al periodo frío.

Durante el periodo cálido, en un $91.8 \%$ de los días la temperatura mínima en el centro de Barcelona ha sido superior, con una diferencia media de temperatura de $1.5^{\circ} \mathrm{C}$. La clase modal es $1.0-2.0^{\circ} \mathrm{C}$, que acumula casi el $45 \%$ de los valores.

Citación: MARTINEZ, M. D. et al. Características de la isla de calor urbana en Barcelona (NE España) en el periodo 20062017. En: Libro de proceedings, CTV 2018. XII Congreso Internacional Ciudad y Territorio Virtual. "Ciudades y Territorios Inteligentes". UNCuyo, Mendoza, 5-7 septiembre 2018. Barcelona: CPSV, 2018, p. 369-380. 
Figura 5. Histograma de los valores de $\Delta T_{\min }$ a escala estacional
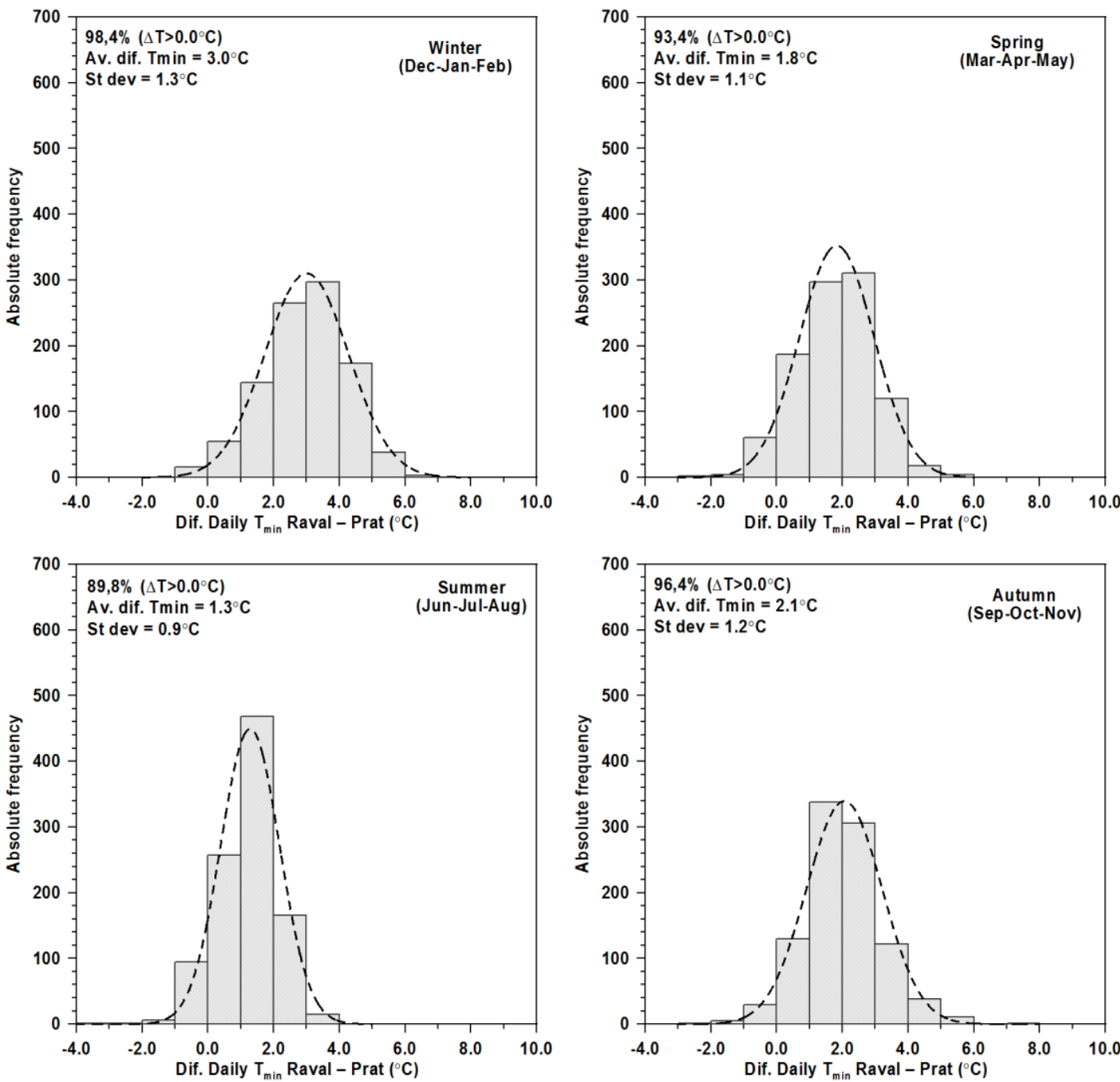

Fuente: Elaboración propia

La Figura 5 muestra los histogramas de los valores de $\Delta T_{\min }$ a escala estacional, en los cuales se observan claramente las diferencias de la isla de calor entre el invierno y el verano.

En invierno, prácticamente la totalidad de las noches (un 98.4\%) son más cálidas en El Raval, con una diferencia media de temperatura de $3.0^{\circ} \mathrm{C}$. La clase modal es la de $3.0-4.0^{\circ} \mathrm{C}$.

En verano, se alcanza la menor proporción de noches más cálidas en El Raval (89.8\%) y la menor diferencia media de temperatura $\left(1.3^{\circ} \mathrm{C}\right)$. La clase modal es la de $1.0-2.0^{\circ} \mathrm{C}$. La forma de los histogramas para otoño y primavera es similar, con una proporción de noches más cálidas en El Raval ligeramente superior en otoño (96.4\% vs $93.4 \%$ ) y una diferencia media de temperatura también algo mayor $\left(2.1^{\circ} \mathrm{C}\right.$ vs $\left.1.8^{\circ} \mathrm{C}\right)$.

En todos los histogramas, la línea de trazos representa la distribución gaussiana que correspondería a los respectivos valores de la media y de la desviación standard. Se observa que, en general, las distribuciones de la intensidad de la isla de calor no se ajustan bien a un modelo gaussiano.

Citación: MARTINEZ, M. D. et al. Características de la isla de calor urbana en Barcelona (NE España) en el periodo 20062017. En: Libro de proceedings, CTV 2018. XII Congreso Internacional Ciudad y Territorio Virtual. "Ciudades y Territorios Inteligentes". UNCuyo, Mendoza, 5-7 septiembre 2018. Barcelona: CPSV, 2018, p. 369-380. 


\subsection{Espectro de potencia de las diferencias de temperatura mínima}

El espectro de potencia de una serie proporciona una estructura fina de sus contenidos espectrales. La Figura 6 muestra, en doble escala logarítmica, el espectro de potencia de la serie de diferencias diarias de temperatura mínima. El espectro de potencia se calcula después de haber eliminado la tendencia lineal de la serie, con el fin de prevenir la aparición de falsas periodicidades. Se observan claramente dos picos de la amplitud espectral para las periodicidades de un año y de 6 meses, lo cual indica que la intensidad de la isla de calor en Barcelona es un fenómeno con periodicidad anual y semianual.

Figura 6. Espectro de potencia de los valores de $\Delta \boldsymbol{T}_{\min }$

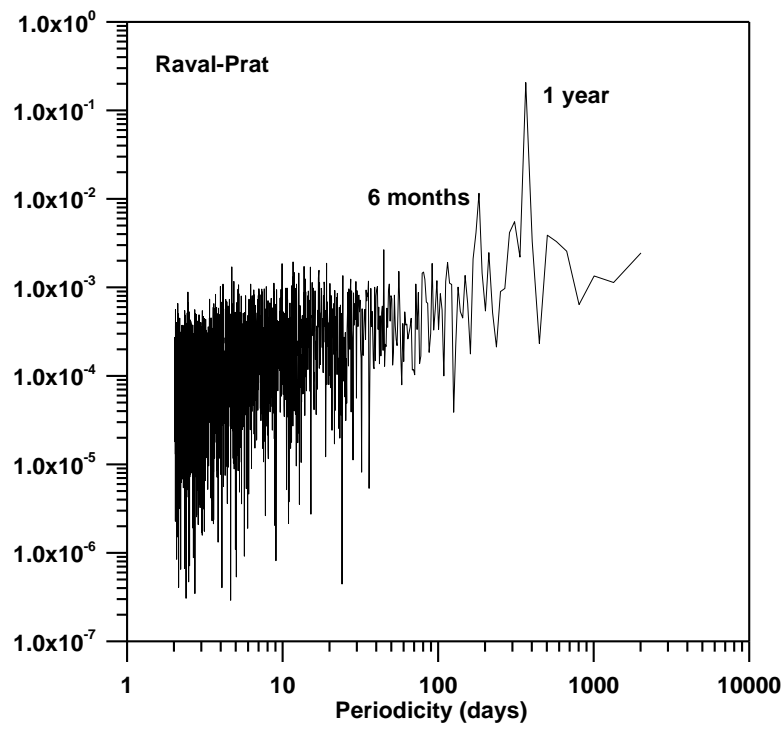

Fuente: Elaboración propia

\subsection{Distribución de las diferencias de temperatura mínima en función del día del año}

En la Figura 7, se representan los más de 4000 valores diarios de $\Delta T_{\min }$ en función del día del año (calendario juliano). Este tipo de representación permite explorar en detalle el comportamiento de la intensidad de la isla de calor a lo largo del año. La nube de puntos se puede ajustar aproximadamente por un polinomio de segundo grado. Esta forma parabólica refleja que la máxima intensidad de la isla de calor corresponde al periodo frío del año. Los mayores valores de $\Delta T_{\min }$ se dan, en general, entre los días 1 y 60 (meses de enero y febrero) y entre los días 300 y 365 (meses de noviembre y diciembre).

Los menores valores positivos de $\Delta T_{\min }$ ocurren entre los días 180 y 240 (meses de julio y agosto). Tal y como ya se ha obtenido en la Sección 3.1, se observa que los valores negativos de $\Delta T_{\min }$, que corresponden a días en que la temperatura mínima ha sido inferior en El Raval, son muy escasos, y son prácticamente inexistentes en los meses de enero y diciembre.

A lo largo de todo el periodo de 11 años estudiado, esta circunstancia solo ha ocurrido 3 veces en el mes de enero y 4 veces en el mes de diciembre. La mayor concentración de estos escasos valores negativos de $\Delta T_{\min }$ se da entre los días 120 y 240 , es decir, en el periodo central del año. 
Figura 7. Distribución de las diferencias de temperatura mínima según el día del año

Fuente: Elaboración propia

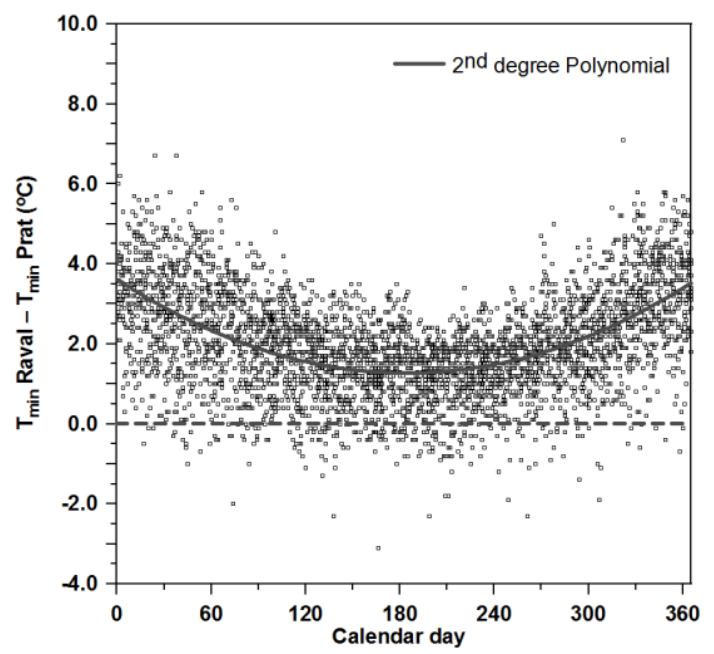

\subsection{Influencia de la velocidad del viento en la intensidad de la isla de calor}

Con el objetivo de explorar el papel de la velocidad del viento como atenuante o moderador de la intensidad de la isla de calor, el rango de los valores diarios de la velocidad del viento registrados en la estación de El Raval, a lo largo de todo el periodo de estudio, se ha dividido en intervalos de $0.2 \mathrm{~m} \cdot \mathrm{s}^{-1}$.

Figura 8. Valor medio (izquierda) y valor máximo (derecha) de $\Delta \boldsymbol{T}_{\min }$, para intervalos de velocidad del viento de $0.2 \mathrm{~m} \cdot \mathrm{s}^{-1}$
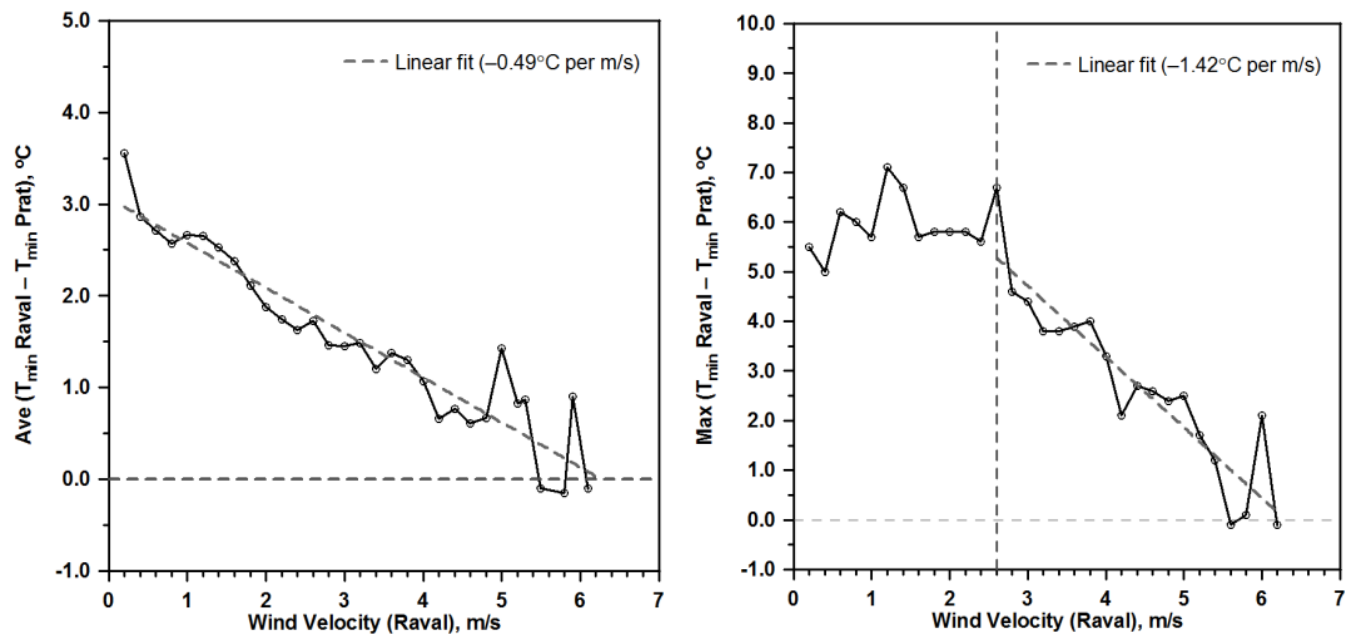

Fuente: Elaboración propia

Cada uno de los valores diarios de $\Delta T_{\min }$ se clasifica en el intervalo que les corresponde, de acuerdo con la velocidad del viento registrada ese día. A continuación, para cada uno de los intervalos de velocidad del viento, se calcula el promedio de los valores diarios de $\Delta T_{\min }$ y se busca el máximo valor de $\Delta T_{\min }$. En la Figura 8, se representan los valores medio y máximo de $\Delta T_{\min }$, para cada intervalo de velocidad del viento. Los resultados muestran una disminución clara del valor medio de $\Delta T_{\min }$ al aumentar la velocidad del viento (Figura 8, izquierda). Si se 
determina la recta que mejor ajusta los puntos, se obtiene que la tasa de disminución sería de $-0.49^{\circ} \mathrm{C}$ por $\mathrm{m} \cdot \mathrm{s}^{-1}$. La evolución de los valores máximos de $\Delta T_{\min }$ (Figura 8 , derecha) presenta algunas fluctuaciones hasta que se alcanza un cierto valor de la velocidad del viento (alrededor de $2.5 \mathrm{~m} \cdot \mathrm{s}^{-1}$ ), a partir del cual el valor máximo de $\Delta T_{\min }$ decrece a un ritmo $-1.42^{\circ} \mathrm{C}$ por $\mathrm{m} \cdot \mathrm{s}^{-1}$ (asumiendo un ajuste lineal). Este resultado sugiere que es necesario superar un cierto valor crítico de la velocidad para que el efecto del viento comience a atenuar los episodios más intensos de la isla de calor.

\section{Conclusiones}

En este trabajo se analiza la intensidad de la isla de calor en Barcelona a lo largo de un periodo de 11 años (octubre 2006-octubre 2017). Para ello, se comparan las temperaturas mínimas diarias registradas en la estación meteorológica El Raval, ubicada en el centro de Barcelona, con las registradas en el observatorio del aeropuerto de Barcelona (El Prat), situado en el área metropolitana. El periodo de estudio proporciona más de 4000 datos diarios de diferencias de temperaturas mínimas entre El Raval y El Prat (Ap).

El análisis de estos datos revela que, en casi el 95\% de los días, la temperatura mínima ha sido más elevada en El Raval que en El Prat (Ap), una proporción que se eleva hasta el $98.4 \%$ en los meses de invierno, durante los cuales la temperatura mínima en el centro de la ciudad es, en promedio, $3.0^{\circ} \mathrm{C}$ superior. La máxima diferencia de temperatura mínima registrada en el periodo analizado es de $7.1^{\circ} \mathrm{C}$ y corresponde al 18 de noviembre de 2007.

Todos los valores extremos de la intensidad de la isla de calor, superiores a $5.0^{\circ} \mathrm{C}$, se han dado durante el periodo frío del año. La escasa proporción de días en los cuales la temperatura mínima ha sido inferior en El Raval corresponde, principalmente, a los meses entre junio y agosto. Estos resultados son coherentes con los obtenidos por Martín-Vide et al. (2015b), que comparan, para el periodo 2004-2013, las temperaturas mínimas en El Prat (Ap) con las del observatorio meteorológico de Barcelona-Vila Olímpica. La mayor intensidad de la isla de calor en invierno, durante la noche o a primeras horas de la mañana, ha sido señalada en numerosos estudios (Montávez et al., 2000; Arnfield, 2003; Liu et al., 2007; Memon et al., 2009; Giannaros and Melas, 2012, entre otros).

Con el fin de explorar el efecto de la velocidad del viento como atenuante o moderador de la intensidad de la isla de calor, se analizan los valores promedio y máximo de las diferencias de temperatura mínima para diferentes clases de velocidad del viento, para intervalos de 0.2 $\mathrm{m} \cdot \mathrm{s}^{-1}$. Los valores medios de las diferencias de temperatura disminuyen claramente al aumentar la velocidad del viento en El Raval. El efecto del viento como moderador de la intensidad de la isla de calor ha sido observado en numerosos estudios (Giannaros and Melas, 2012; Papanastasiou and Kittas, 2012; Founda et al., 2015, entre otros).

En cuanto a la evolución de los valores máximos de las diferencias de temperatura, se observa que es necesario superar un cierto valor crítico de la velocidad para que el efecto del viento comience a atenuar los episodios más intensos de la isla de calor. La existencia de un valor crítico de la velocidad del viento a partir del cual se modera la intensidad de la isla de calor es coherente con los resultados obtenidos en diversos estudios (Morris et al., 2001; László et al., 2016, entre otros).

Citación: MARTINEZ, M. D. et al. Características de la isla de calor urbana en Barcelona (NE España) en el periodo 20062017. En: Libro de proceedings, CTV 2018. XII Congreso Internacional Ciudad y Territorio Virtual. "Ciudades y Territorios Inteligentes". UNCuyo, Mendoza, 5-7 septiembre 2018. Barcelona: CPSV, 2018, p. 369-380. 


\section{Bibliografía}

ARELLANO, B.; ROCA, J. Identifying urban heat island: the Barcelona case. En: International Conference Virtual City and Territory - 11th Congress Virtual City and Territory, 798-812, 2016.

ARNFIELD, A. J. Two decades of urban climate research: a review of turbulence, exchanges of energy and water, and the urban heat island. En: International Journal of Climatology, 23, 1-26, 2003.

FIGUEROLA, P. I.; NICOLÁS, A.; MAZZEO, N. A. Urban-rural temperature differences in Buenos Aires. En: International Journal of Climatology, 18, 1709-1723, 1998.

FORTUNIAK, K.; KLYSIK, K.; WIBIG, J. Urban-rural contrasts of meteorological parameters in Lódz. En: Theoretical and Applied Climatology, 84, 91-101, 2006.

FOUNDA, D.; PIERROS, F.; PETRAKIS, M.; ZEREFOS, C. Interdecadal variations and trends of the Urban Heat Island in Athens (Greece) and its response to heat waves. En: Atmospheric Research, 161-162, 1-13, 2015.

GIANNAROS, T. M.; MELAS, D. Study of the urban heat island in a coastal Mediterranean City: The case study of Thessaloniki, Greece. En: Atmospheric Research, 118, 103-120, 2012.

HOWARD, L. Climate of London deduced from meteorological observations. 3rd ed. (3 Vols.) Harvey \& Darton, London, 1833.

LÁSZLÓ, E.; BOTTYÁN, Z.; SZEGEDI, S. Long-term changes of meteorological conditions of urban heat island development in the region of Debrecen, Hungary. En: Theoretical and Applied Climatology, 124, 365-373, 2016.

LIU, X.; JI, C.; ZHONG, J.; JIANG, X.; ZHENG, Z. Temporal characteristics of the Beijing urban heat island. En: Theoretical and Applied Climatology, 87, 213-221, 2007.

MAKAR, P. A.; GRAVEL S.; CHIRKOV, V.; STRAWBRIDGE, K. B.; FROUDE, F.; ARNOLD, J.; BROOK, J. Heat flux, urban properties, and regional weather. En: Atmospheric Environment, 40: 2750-2766, 2006.

MARTÍN-VIDE, J.; SARRICOLEA, P.; MORENO-GARCÍA, M. C. On the definition of urban heat island intensity: the "rural reference". En: Frontiers in Earth Science, 3, 1-3, $2015 \mathrm{a}$.

MARTÍN-VIDE, J.; CORDOBILLA, M. J.; MORENO-GARCÍA, M. C. La isla de calor en el área metropolitana de Barcelona y la adaptación al cambio climático. METROBS-2015. Direcció de Serveis Ambientals, Área Metropolitana de Barcelona, AMB, 101 pp. 2015b.

MEMON, R.A.; LEUNG, D.Y.C.; LIU, Ch.-H. An investigation of urban heat island intensity (UHII) as an indicator of urban heating. En: Atmospheric Research, 94, 491-500, 2009. 
MONTÁVEZ, J. P.; RODRÍGUEZ, A.; JIMÉNEZ, J. I. A study of the urban heat island of Granada, Spain. En: International Journal of Climatology, 20, 899-911, 2000.

MORENO-GARCIA, M. C. Intensity and form of the urban heat island in Barcelona. En: International Journal of Climatology, 14, 705-710, 1994.

MORRIS, C. J. G. \& SIMMONDS, I. Associations between varying magnitudes of the urban heat island and the synoptic climatology in Melbourne, Australia. En: International Journal of Climatology, 20, 1931-1954, 2000.

MORRIS, C. J. G.; SIMMONDS, I. \& PLUMMER, N. Quantification of the Influences of Wind and Cloud on the Nocturnal Urban Heat Island of a Large City. En: Journal of Applied Meteorology, 40, 169-182, 2001.

OKE, T. R. The energetic basis of the urban heat island. En: Quarterly Journal of the Royal Meteorological Society, 108: 1-24, 1982.

PAPANASTASIOU, D. K. \& KITTAS, C. Maximum urban heat island intensity in a medium-sized coastal Mediterranean city. En: Theoretical and Applied Climatology, 107, 407-416, 2012.

WANNER, H. \& HERTIG, J. Studies of urban climates and air pollution in Switzerland. En: Journal of Applied Meteorology, 23:1614-1625, 1984.

Citación: MARTINEZ, M. D. et al. Características de la isla de calor urbana en Barcelona (NE España) en el periodo 20062017. En: Libro de proceedings, CTV 2018. XII Congreso Internacional Ciudad y Territorio Virtual. "Ciudades y Territorios Inteligentes". UNCuyo, Mendoza, 5-7 septiembre 2018. Barcelona: CPSV, 2018, p. 369-380. 